\title{
Chapter 7 \\ The Legacy of Rolf Hagedorn: Statistical Bootstrap and Ultimate Temperature
}

\author{
Krzysztof Redlich and Helmut Satz
}

\begin{abstract}
In the latter half of the last century, it became evident that there exists an ever increasing number of different states of the so-called elementary particles. The usual reductionist approach to this problem was to search for a simpler infrastructure, culminating in the formulation of the quark model and quantum chromodynamics. In a complementary, completely novel approach, Hagedorn suggested that the mass distribution of the produced particles follows a self-similar composition pattern, predicting an unbounded number of states of increasing mass. He then concluded that such a growth would lead to a limiting temperature for strongly interacting matter. We discuss the conceptual basis for this approach, its relation to critical behavior, and its subsequent applications in different areas of high energy physics.
\end{abstract}

A prophet is not without honour, but in his own country. The New Testament, Mark 6,4.

\subsection{Rolf Hagedorn}

The development of physics is the achievement of physicists, of humans, persisting against often considerable odds. Even in physics, fashion rather than fact frequently determines judgment and recognition.

When Rolf Hagedorn (Fig. 7.1) carried out his main work, now quite generally recognized as truly pioneering, much of the theoretical community not only ignored it, but even considered it to be nonsense. "Hagedorn ist ein Narr", he is a fool, was a summary of many leading German theorists of his time. When in the 1990s

K. Redlich ( $\square)$

Institute of Theoretical Physics, University Wroclaw, Wroclaw, Poland

H. Satz $(\bowtie)$

Fakultät für Physik, Universität Bielefeld, Bielefeld, Germany 


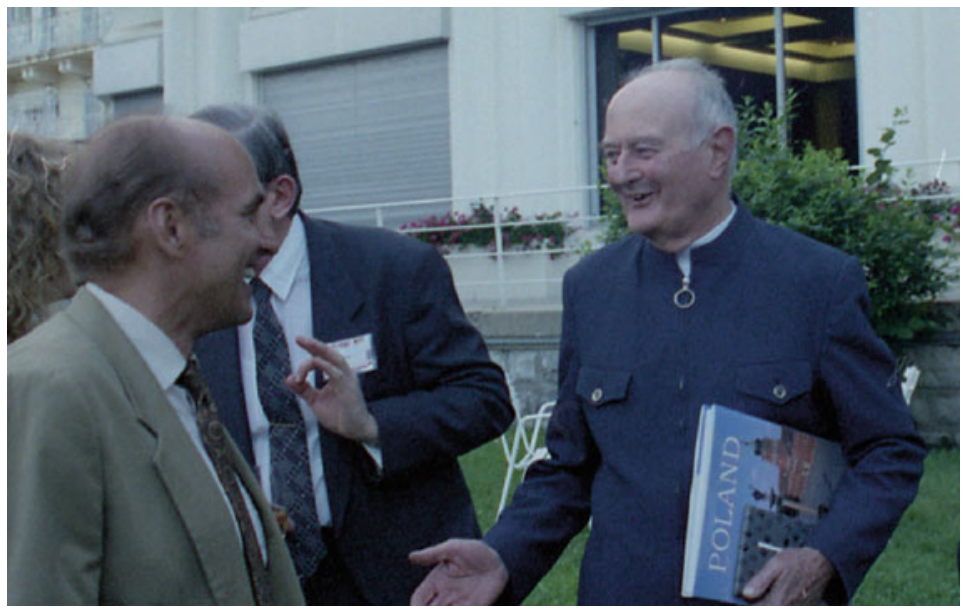

Fig. 7.1 Rolf Hagedorn (on right) in conversation with Helmut Satz (on left), 30 June 1994. Hagedorn holds a birthday gift from Krzysztof Redlich. Image credit: CERN Image 1994-06-64022

the question was brought up whether he could be proposed for the Max Planck Medal, the highest honor of the German physics community, even then, when his achievements were already known world-wide, the answer was still "proposed, yes...."

At the time Hagedorn carried out his seminal research, much of theoretical physics was ideologically fixed on "causality, unitarity, Poincaré invariance": from these three concepts, from axiomatic quantum field theory, all that is relevant to physics must arise. Those who thought that science should progress instead by comparison to experiment were derogated as "fitters and plotters". Galileo was almost forgotten.... Nevertheless, one of the great Austrian theorists of the time, Walter Thirring, himself probably closer to the fundamentalists, noted: "If you want to do something really new, you first have to have a new idea". Hagedorn did.

He had a number of odds to overcome. He had studied physics in Göttingen under Richard Becker, where he developed a life-long love for thermodynamics. When he took a position at CERN, shortly after completing his doctorate, it was to perform calculations for the planning and construction of the proton synchrotron. When that was finished, he shifted to the study of multihadron production in proton-proton collisions and to modeling the results of these reactions. It took a while before various members of the community, including some of the CERN Theory Division, were willing to accept the significance of his work. This was not made easier by Hagedorn's strongly focused region of interest, but eventually it became generally recognized that here was someone who, in this perhaps similar to John Bell, was developing truly novel ideas which at first sight seemed quite specific, but which eventually turned out to have a lasting impact also on physics well outside its region of origin. 
We find that Rolf Hagedorn's work centers on two themes:

- the statistical bootstrap model, a self-similar scheme for the composition and decay of hadrons and their resonances; for Hagedorn, these were the "fireballs".

- the application of the resulting resonance spectrum in an ideal gas containing all possible hadrons and hadron resonances, and to the construction of hadron production models based on such a thermal input.

We will address these topics in the first two sections, and then turn to their roles both in the thermodynamics of strongly interacting matter and in the description of hadron production in elementary as well as nuclear collisions. Our aim here is to provide a general overview of Hagedorn's scientific achievements. Some of what we will say transcends Hagedorn's life. But then, to paraphrase Shakespeare, we have come to praise Hagedorn, not to bury him; we want to show that his ideas are still important and very much alive.

\subsection{The Statistical Bootstrap}

Around 1950, the physics world still seemed in order for those looking for the ultimate constituents of matter in the universe. Dalton's atoms had been found to be not really atoms, indivisible; Rutherford's model of the atom had made them little planetary systems, with the nucleus as the sun and the electrons as encircling planets. The nuclei in turn consisted of positively charged protons and neutral neutrons as the essential mass carriers. With an equal number of protons and electrons, the resulting atoms were electrically neutral, and the states obtained by considering the different possible nucleus compositions reproduced the periodic table of elements. So for a short time, the Greek dream of obtaining the entire complex world by combining three simple elementary particles in different ways seemed finally feasible: protons, neutrons and electrons were the building blocks of our universe.

But there were those who rediscovered an old problem, first formulated by the Roman philosopher Lucretius: if your elementary particles, in our case the protons and neutrons, have a size and a mass, as both evidently did, it was natural to ask what they are made of. An obvious way to find out is to hit them against each other and look at the pieces. And it turned out that there were lots of fragments, the more, the harder the collision. But they were not really pieces, since the debris found after a proton-proton collision still also contained the two initial protons. Moreover, the additional fragments, mesons and baryons, were in almost all ways as elementary as protons and neutrons. The study of such collisions was taken up by more and more laboratories and at ever higher collision energies. As a consequence, the number of different "elementary" particles grew by leaps and bounds, from tens to twenties to hundreds. The latest compilation of the Particle Data Group contains over a thousand. 


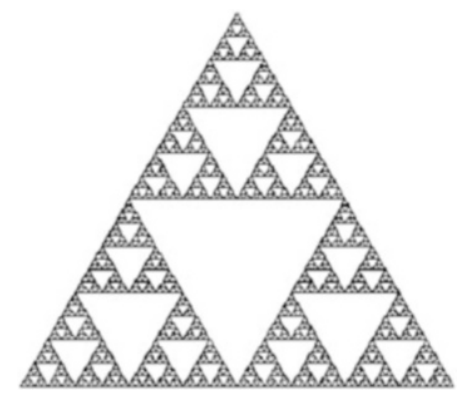

Fig. 7.2 The Sierpinski triangle

Let us, however, return to the time when physics was confronted by all those elementary particles, challenging its practitioners to find a way out. At this point, in the mid 1960s, Rolf Hagedorn came up with a truly novel idea [1-6]. He was not so much worried about the specific properties of the particles. He just imagined that a heavy particle was somehow composed out of lighter ones, and these again in turn of still lighter ones, and so on, until one reached the pion as the lightest hadron. And by combining heavy ones, you would get still heavier ones, again: and so on. The crucial input was that the composition law should be the same at each stage. Today we call that self-similarity, and it had been around in various forms for many years. A particularly elegant formulation was written a 100 years before Hagedorn by the English mathematician Augustus de Morgan, the first president of the London Mathematical Society:

Great fleas have little fleas upon their backs to bite'em, and little fleas have lesser still, and so ad infinitum.

And the great fleas themselves, in turn, have greater fleas to go on, while these again have greater still, and greater still, and so on.

Hagedorn proposed that "a fireball consists of fireballs, which in turn consist of fireballs, and so on...." The concept later reappeared in various forms in geometry; in 1915, it led to the celebrated triangle, see Fig. 7.2 devised by the Polish mathematician Wacław Sierpinski: "a triangle consists of triangles, which in turn consist of triangles, and so on...," in the words of Hagedorn. Still later, shortly after Hagedorn's proposal, the French mathematician Benoit Mandelbroit initiated the study of such fractal behavior as a new field of mathematics.

Hagedorn had recalled a similar problem in number theory: how many ways are there of decomposing an integer into integers? This was something already addressed in 1753 by Leonhard Euler, and more than a century later by the mathematician E. Schröder in Germany. Finally G.H. Hardy and S. Ramanujan in England provided an asymptotic solution [7]. Let us here, however, consider a simplified, easily solvable version of the problem [8], in which we count all possible 
different ordered arrangements $p(n)$ of an integer $n$. So we have

$$
\begin{array}{lr}
1=1 & p(1)=1=2^{n-1} \\
2=2,1+1 & p(2)=2=2^{n-1} \\
& p(3)=4=2^{n-1} \\
3=3,2+1,1+2,1+1+1 & \\
4=4,3+1,1+3,2+2,2+1+1,1+2+1,1+1+2,1+1+1+1 \\
p(4)=8=2^{n-1}
\end{array}
$$

and so on. In other words, there are

$$
p(n)=2^{n-1}=\frac{1}{2} e^{n \ln 2}
$$

ways of partitioning an integer $n$ into ordered partitions: $p(n)$ grows exponentially in $n$. In this particular case, the solution could be found simply by induction. But there is another way of reaching it, more in line with Hagedorn's thinking: "large integers consist of smaller integers, which in turn consist of still smaller integers, and so on...." This can be formulated as an equation,

$$
\rho(n)=\delta(n-1)+\sum_{k=2}^{n} \frac{1}{k !} \prod_{i=1}^{k} \rho\left(n_{i}\right) \delta\left(\Sigma_{i} n_{i}-n\right) .
$$

It is quite evident here that the form of the partition number $\rho(n)$ is determined by a convolution of many similar partitions of smaller $n$. The solution of the equation is in fact just the number of partitions of $n$ that we had obtained above,

$$
\rho(n)=z p(n)
$$

up to a normalization constant of order unity (for the present case, it turns out that $z \simeq$ 1.25). For Hagedorn, Eq. (7.2) expressed the idea that the structure of $\rho(n)$ was determined by the structure of $\rho(n)$-we now call this self-similar. He instead thought of the legendary Baron von Münchhausen, who had extracted himself from a swamp by pulling on his own bootstraps. So for him, Eq. (7.2) became his bootstrap equation.

The problem Hagedorn had in mind was, of course, considerably more complex. His heavy resonance was not simply a sum of lighter ones at rest, but it was a system of lighter resonances in motion, with the requirement that the total energy of this system added up to the mass of the heavy one. And similarly, the masses of the lighter ones were the result of still lighter ones in motion. The bootstrap equation for such a situation becomes

$$
\rho\left(m, V_{0}\right)=\delta\left(m-m_{0}\right)+\sum_{N} \frac{1}{N !}\left[\frac{V_{0}}{(2 \pi)^{3}}\right]^{N-1} \int \prod_{i=1}^{N}\left[d m_{i} \rho\left(m_{i}\right) d^{3} p_{i}\right] \delta^{4}\left(\Sigma_{i} p_{i}-p\right),
$$

where the first term corresponds to the case of just one lightest possible particle, a "pion". The factor $V_{0}$, the so-called composition volume, specified the size of the overall system, an intrinsic fireball size. Since the mass of any resonance in the composition chain is thus determined by the sum over phase spaces containing 
lighter ones, whose mass is specified in the same way, Hagedorn called this form of bootstrap "statistical".

After a number of numerical attempts by others, W. Nahm [9] solved the statistical bootstrap equation analytically, obtaining

$$
\rho\left(m, V_{0}\right)=\text { const. } m^{-3} \exp \left\{m / T_{\mathrm{H}}\right\} .
$$

So even though the partitioning now was not just additive in masses, but included the kinetic energy of the moving constituents, the increase was again exponential in mass. The coefficient of the increase, $T_{\mathrm{H}}{ }^{-1}$, is determined by the equation

$$
\frac{V_{0} T_{\mathrm{H}}{ }^{3}}{2 \pi^{2}}\left(m_{0} / T_{\mathrm{H}}\right)^{2} K_{2}\left(m_{0} / T_{\mathrm{H}}\right)=2 \ln 2-1,
$$

in terms of two parameters $V_{0}$ and $m_{0}$. Hagedorn assumed that the composition volume $V_{0}$, specifying the intrinsic range of strong interactions, was determined by the inverse pion mass as scale, $V_{0} \simeq(4 \pi / 3) m_{\pi}^{-3}$. This leads to a scale factor $T_{\mathrm{H}} \simeq 150 \mathrm{MeV}$. It should be emphasized, however, that this is just one possible way to proceed. In the limit $m_{0} \rightarrow 0$, Eq. (7.6) gives

$$
T_{\mathrm{H}}=\left[\pi^{2}(2 \ln 2-1)\right]^{1 / 3} V_{0}^{-1 / 3} \simeq 1 / r_{\mathrm{h}},
$$

where $V_{0}=(4 \pi / 3) r_{\mathrm{h}}^{3}$ and $r_{\mathrm{h}}$ denotes the range of strong interactions. With $r_{\mathrm{h}} \simeq$ $1 \mathrm{fm}$, we thus have $T_{\mathrm{H}} \simeq 200 \mathrm{MeV}$. From this it is evident that the exponential increase persists also in the chiral limit $m_{\pi} \rightarrow 0$ and is in fact only weakly dependent on $m_{0}$, provided the strong interaction scale $V_{0}$ is kept fixed.

The weights $\rho(m)$ determine the composition as well as the decay of "resonances", of fireballs. The basis of the entire formalism, the self-similarity postulate-here in the form of the statistical bootstrap condition-results in an unending sequence of ever-heavier fireballs and in an exponentially growing number of different states of a given mass $m$.

Before we turn to the implications of such a pattern in thermodynamics, we note that not long after Hagedorn's seminal paper, it was found that a rather different approach, the dual resonance model [10-12], see Chap. 8, led to very much the same exponential increase in the number of states. In this model, any scattering amplitude, from an initial two to a final hadrons, was assumed to be determined by the resonance poles in the different kinematic channels. This resulted structurally again in a partition problem of the same type, and again the solution was that the number of possible resonance states of mass $m$ must grow exponentially in $m$, with an inverse scale factor of the same size as obtained above, some $200 \mathrm{MeV}$. Needless to say, this unexpected support from the forefront of theoretical hadron dynamics considerably enhanced the interest in Hagedorn's work. 


\subsection{The Limiting Temperature of Hadronic Matter}

Consider a relativistic ideal gas of identical neutral scalar particles of mass $m_{0}$ contained in a box of volume $V$, assuming Boltzmann statistics. The grand canonical partition function of this system is given by

$$
\mathscr{Z}(T, V)=\sum_{N} \frac{1}{N !}\left[\frac{V}{(2 \pi)^{3}} \int d^{3} p \exp \left\{-\sqrt{p^{2}+m_{0}^{2}} / T\right\}\right]^{N},
$$

leading to

$$
\ln \mathscr{Z}(T, V)=\frac{V T m_{0}^{2}}{2 \pi^{2}} K_{2}\left(m_{0} / T\right) .
$$

For temperatures $T \gg m_{0}$, the energy, and the particle density of the system become, respectively

$$
\varepsilon(T)=-\frac{1}{V} \frac{\partial \ln \mathscr{Z}(T, V)}{\partial(1 / T)} \simeq \frac{3}{\pi^{2}} T^{4}, \quad n(T)=\frac{\partial \ln \mathscr{Z}(T, V)}{\partial V} \simeq \frac{1}{\pi^{2}} T^{3},
$$

and so the average energy per particle is given by

$$
\frac{E}{N} \simeq 3 T
$$

The important feature to learn from these relations is that, in the case of an ideal gas of one species of elementary particles, an increase of the energy of the system has three consequences. It leads to:

- a higher temperature,

- more constituents, and

- more energetic constituents.

If we now consider an interacting gas of such basic hadrons and postulate that the essential form of the interaction is resonance formation, then we can approximate the interacting medium as a non-interacting gas of all possible resonance species $[13,14]$. The partition function of this resonance gas is

$$
\ln \mathscr{Z}(T, V)=\sum_{i} \frac{V T m_{i}^{2}}{2 \pi^{2}} \rho\left(m_{i}\right) K_{2}\left(m_{i} / T\right)
$$

where the sum begins with the stable ground state $m_{0}$ and then includes the possible resonances $m_{i}, i=1,2, \ldots$ with weights $\rho\left(m_{i}\right)$ relative to $m_{0}$. Clearly the crucial question here is how to specify $\rho\left(m_{i}\right)$, that is how many states there are of mass $m_{i}$. It is only at this point that hadron dynamics enters, and it is here that Hagedorn introduced the result obtained in his statistical bootstrap model. 
As we had seen above in Eq. (7.5), the density of states then increases exponentially in $m$, with a coefficient $T_{\mathrm{H}}{ }^{-1}$ determined by Eq. (7.6) in terms of two parameters $V_{0}$ and $m_{0}$. If we replace the sum in the resonance gas partition function Eq. (7.12) by an integral and insert the exponentially growing mass spectrum Eqs. (7.5) and (7.12) becomes

$$
\ln \mathscr{Z}(T, V) \simeq \frac{V T}{2 \pi^{2}} \int d m m^{2} \rho\left(m_{i}\right) K_{2}\left(m_{i} / T\right) \sim V\left[\frac{T}{2 \pi}\right]^{3 / 2} \int \frac{d m}{m^{3 / 2}} e^{-\left[\frac{m}{T}-\frac{m}{T_{\mathrm{H}}}\right]}
$$

Evidently, the result is divergent for all $T>T_{\mathrm{H}}$ : in other words, $T_{\mathrm{H}}$ is the highest possible temperature of hadronic matter. Moreover, if we compare such a system with the ideal gas of only basic particles (a "pion" gas), we find:

$$
\begin{array}{lll}
\text { pion gas } & & \text { resonance gas } \\
n_{\pi} \sim \varepsilon^{3 / 4} & & n_{\text {res }} \sim \varepsilon \\
\omega_{\pi} \sim \varepsilon^{1 / 4} & & \omega_{\text {res }} \sim \text { const. }
\end{array}
$$

Here $n$ denotes the average number density of constituents, $\omega$ the average energy of a constituent. In contrast to the pion gas, an increase of energy now leads to

- a fixed temperature limit, $T \rightarrow T_{\mathrm{H}}$,

- the momenta of the constituents do not continue to increase, and

- more and more species of ever heavier particles appear.

We thus obtain a new, non-kinetic way to use energy, increasing the number of species and their masses, not the momentum per particle. Temperature is a measure of the momentum of the constituents, and if that cannot continue to increase, there is a highest possible, a "limiting" temperature for hadronic systems.

Hagedorn originally interpreted $T_{\mathrm{H}}$ as the ultimate temperature of strongly interacting matter. It is clear today that $T_{\mathrm{H}}$ signals the transition from hadronic matter to a quark-gluon plasma. Hadron physics alone can only specify its inherent limit; to go beyond this limit, we need more information: we need QCD.

As seen in Eq. (7.5), the solution of the statistical bootstrap equation has the general form

$$
\rho\left(m, V_{0}\right) \sim m^{-a} \exp \left(m / T_{\mathrm{H}}\right),
$$

with some constant $a$; the exact solution of Eq. (7.4) by Nahm gave $a=3$. It is possible, however, to consider variations of the bootstrap model which lead to different $a$, but always retain the exponential increase in $m$. While the exponential form makes $T_{\mathrm{H}}$ the upper limit of permissible temperatures, the power law coefficient $a$ determines the behavior of the system at $T=T_{\mathrm{H}}$. For $a=3$, the partition function Eq. (7.13) itself exists at that point, while the energy density as first derivative in temperature diverges there. This is what made Hagedorn conclude 
that $T_{\mathrm{H}}$ is indeed the highest possible temperature of matter: it would require an infinite energy to reach it.

Only a few years later it was, however, pointed out by N. Cabibbo and G. Parisi [15] that larger $a$ shifted the divergence at $T=T_{\mathrm{H}}$ to ever higher derivatives. In particular, for $4>a>3$, the energy density would remain finite at that point, shifting the divergence to the specific heat as next higher derivative. Such critical behavior was in fact quite conventional in thermodynamics: it signaled a phase transition leading to the onset of a new state of matter. By that time, the quark model and quantum chromodynamics as fundamental theory of strong interactions had appeared and suggested the existence of a quark-gluon plasma as the relevant state of matter at extreme temperature or density. It was therefore natural to interpret the Hagedorn temperature $T_{\mathrm{H}}$ as the critical transition temperature from hadronic matter to such a plasma. This interpretation is moreover corroborated by a calculation of the critical exponents [16] governing the singular behavior of the resonance gas thermodynamics based on a spectrum of the form Eq. (7.14).

It should be noted, however, that in some sense $T_{\mathrm{H}}$ did remain the highest possible temperature of matter as we know it. Our matter exists in the physical vacuum and is constructed out of fundamental building blocks which in turn have an independent existence in this vacuum. Our matter ultimately consists of and can be broken up into nucleons; we can isolate and study a single nucleon. The quarkgluon plasma, on the other hand, has its own ground state, distinct from the physical vacuum, and its constituents can exist only in a dense medium of other quarks-we can never isolate and study a single quark.

That does not mean, however, that quarks are eternally confined to a given part of space. Let us start with atomic matter and compress that to form nuclear matter, as it exists in heavy nuclei. At this stage, we have nucleons existing in the physical vacuum. Each nucleon consists of three quarks, and they are confined to remain close to each other; there is no way to break up a given nucleon into its quark constituents. But if we continue to compress, then eventually the nucleons will penetrate each other, until we reach a dense medium of quarks. Now each quark finds in its immediate neighborhood many other quarks besides those which were with it in the nucleon stage. It is therefore no longer possible to partition quarks into nucleons; the medium consists of unbound quarks, whose interaction becomes ever weaker with increasing density, approaching the limit of asymptotic freedom predicted by QCD. Any quark can now move freely throughout the medium: we have quark liberation through swarm formation. Wherever a quark goes, there are many other quarks nearby. The transition from atomic to quark matter is schematically illustrated in Fig. 7.3.

We have here considered quark matter formation through the compression of cold nuclear matter. A similar effect is obtained if we heat a meson gas; with increasing temperature, collisions and pair production lead to an ever denser medium of mesons. And according to Hagedorn, also of ever heavier mesons of an increasing degeneracy. For Hagedorn, the fireballs were point like, so that the overlap we had just noted simply does not occur. In the real world, however, they do have hadronic size, so that they will in fact interpenetrate and overlap before the divergence of the Hagedorn resonance gas occurs [17]. Hence now again there will be a transition 
(a)

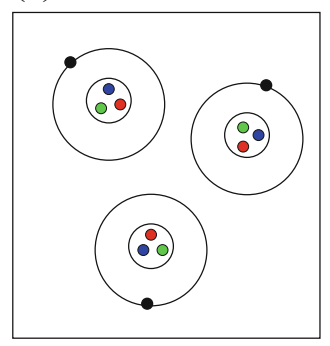

(b)

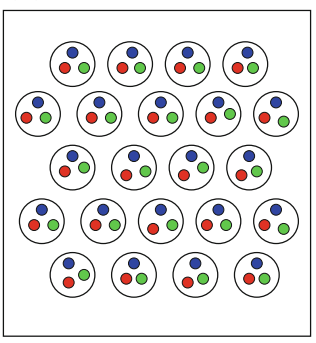

(c)

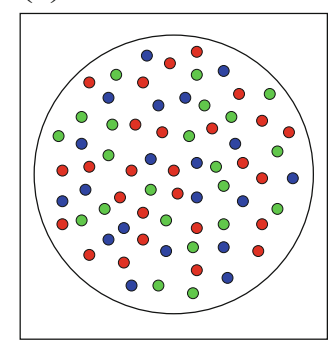

Fig. 7.3 Schematic view of matter for increasing density, from atomic (a) to nuclear (b) and then to quark matter (c)

from resonance gas to a quark-gluon plasma, now formed by the liberation of the quarks and gluons making up the resonances.

At this point, it seems worthwhile to note an even earlier approach leading to a limiting temperature for hadronic matter. More than a decade before Hagedorn, I.Ya. Pomeranchuk [18] pointed out that a crucial feature of hadrons is their size, and hence the density of any hadronic medium is limited by volume restriction: each hadron must have its own volume to exist, and once the density reaches the dense packing limit, it's the end for hadronic matter. This simply led to a temperature limit, and for an ideal gas of pions of $1 \mathrm{fm}$ radius, the resulting temperature was again around $200 \mathrm{MeV}$. Nevertheless, these early results remained largely unnoticed until the work of Hagedorn.

Such geometric considerations do, however, lead even further. If hadrons are allowed to interpenetrate, to overlap, then percolation theory predicts two different states of matter [19, 20]: hadronic matter, consisting of isolated hadrons or finite hadronic clusters, and a medium formed as an infinite sized cluster of overlapping hadrons. The transition from one to the other now becomes a genuine critical phenomenon, occurring at a critical value of the hadron density.

We thus conclude that the pioneering work of Rolf Hagedorn opened up the field of critical behavior in strong interaction physics, a field in which still today much is determined by his ideas. On a more theoretical level, the continuation of such studies was provided by finite temperature lattice $\mathrm{QCD}$, and on the more experimental side, by resonance gas analysis of the hadron abundances in high energy collisions. In both cases, it was found that the observed behavior was essentially that predicted by Hagedorn's ideas.

\subsection{Resonance Gas and QCD Thermodynamics}

With the formulation of Quantum Chromodynamics (QCD) as a theoretical framework for the strong interaction force among elementary particles it became clear that the appearance of the ultimate Hagedorn's temperature $T_{\mathrm{H}}$, signals indeed 
the transition from the hadronic phase to a new phase of strongly interacting matter, the quark-gluon plasma (QGP) [21] (and reference therein). As QCD is an asymptotically free theory, the interaction between quarks and gluons vanishes logarithmically with increasing temperature, thus at very high temperatures the QGP effectively behaves like an ideal gas of quarks and gluons.

Today we have detailed information, obtained from numerical calculations in the framework of finite temperature lattice Quantum Chromodynamics [22, 23], about the thermodynamics of hot and dense matter. We know the transition temperature to the QGP and the temperature dependence of basic bulk thermodynamic observables such as the energy density and the pressure [24, 25]. We also begin to have results on fluctuations and correlations of conserved charges [26-28].

The recent increase in numerical accuracy of lattice QCD calculations and their extrapolation to the continuum limit, makes it possible to confront the fundamental results of QCD with Hagedorn's concepts [2,6], which provide a theoretical scenario for the thermodynamics of strongly interacting hadronic matter [28-30].

In particular, the equation of state calculated on the lattice at vanishing and finite chemical potential, and restricted to the confined hadronic phase, can be directly compared to that obtained from the partition function Eq. (7.13) of the hadron resonance gas, using the form Eq. (7.14) introduced by Hagedorn for a continuum mass spectrum. Alternatively, as a first approximation, one can also consider a discrete mass spectrum which accounts for all experimentally known hadrons and resonances. In this case the continuum partition function of the Hagedorn model is expressed by Eq. (7.12) with $\rho\left(m_{i}\right)$ replaced by the spin degeneracy factor of the $i^{\text {th }}$ hadron, with the summation taken over all known resonance species listed by the Particle Data Group [31].

With the above assumption on the dynamics and the mass spectrum, the resonance gas partition function introduced by Hagedorn [2, 6], can be calculated exactly and expressed as a sum of one-particle partition functions $Z_{i}^{1}$ of all hadrons and resonances,

$$
\ln Z(T, V)=\sum_{i} Z_{i}^{1}(T, V)
$$

For particles of mass $m_{i}$ and spin degeneracy factor $g_{i}$, the one-particle partition function $Z_{i}^{1}$, in the Boltzmann approximation, reads

$$
Z_{i}^{1}(T, V)=g_{i} \frac{V T m_{i}^{2}}{2 \pi^{2}} K_{2}\left(m_{i} / T\right)
$$

Due to the factorization of the partition function in Eq. (7.15), the energy density and the pressure of the Hagedorn resonance gas with a discrete mass spectrum, can also be expressed as a sum over single particle contributions

$$
\varepsilon=\sum_{i} \varepsilon_{i}^{1}, \quad P=\sum_{i} P_{i}^{1}
$$


Fig. 7.4 The normalized pressure $P(T)$, the energy density $\varepsilon(T)$ and the entropy density $s(T)$ obtained in lattice QCD calculations as a function of temperature. The dark lines show predictions of the Hagedorn resonance gas for a discrete mass spectrum, Eqs. (7.17)-(7.20). The lattice results are from [25]

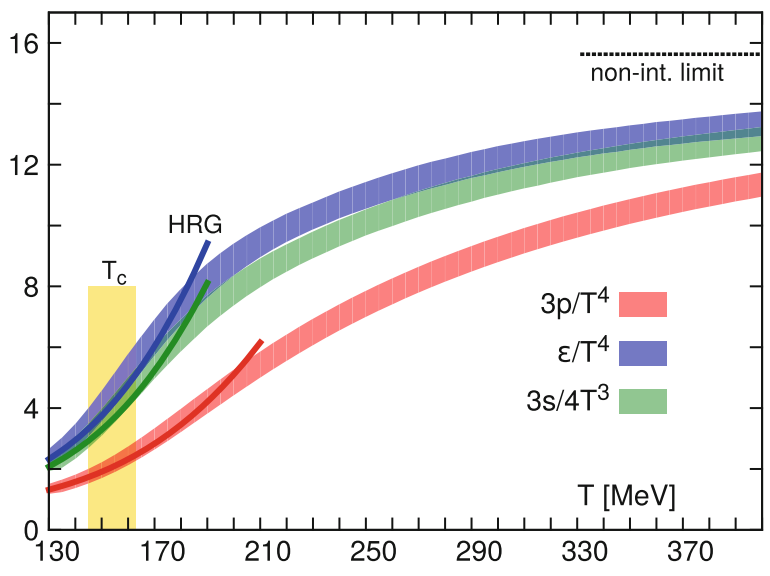

with

$$
\begin{aligned}
& \frac{\varepsilon_{i}^{1}}{T^{4}}=\frac{g_{i}}{2 \pi^{2}}\left(\frac{m_{i}}{T}\right)^{3}\left[\frac{3 K_{2}\left(\beta m_{i}\right)}{\beta m}+K_{1}\left(\beta m_{i}\right)\right] \\
& \frac{P_{i}^{1}}{T^{4}}=\frac{g_{i}}{2 \pi^{2}}\left(\frac{m_{i}}{T}\right)^{3} K_{2}\left(\beta m_{i}\right),
\end{aligned}
$$

where $\beta=1 / T$ and $K_{1}$ and $K_{2}$ are modified Bessel functions. At vanishing chemical potentials and at finite temperature, the energy density $\varepsilon$, the entropy density $s$ and the pressure $P$, are connected through the thermodynamic relation,

$$
\varepsilon=-P+s T \text {. }
$$

Summing up in Eq. (7.17), the contributions from experimentally known hadronic states; constitute the resonance gas [2, 6] (for a review, see e.g. [32]) for the thermodynamics of the hadronic phase of QCD. Taking e.g. contribution of all mesonic and baryonic resonances with masses up to $1.8 \mathrm{GeV}$ and $2.0 \mathrm{GeV}$, respectively, amounts to 1,026 resonances.

The crucial question thus is, if the equation of state of hadronic matter introduced by Hagedorn can describe the corresponding results obtained from QCD within lattice approach.

In Fig. 7.4 we show the temperature dependence of the energy density, pressure and the entropy density obtained recently in lattice QCD studies with physical masses of up, down and strange quarks [25]. The bands in lattice QCD results indicate error bars due to extrapolation to the continuum limit. The vertical band marks the temperature, $T_{c}=(154 \pm 9) \mathrm{MeV}$, which within an error, is the crossover temperature from a hadronic phase to a quark-gluon plasma [33]. These QCD results are compared in Fig. 7.4 to the Hagedorn resonance gas model formulated for a discrete mass spectrum in Eqs. (7.17) and (7.20). 
There is a clear coincidence of the Hagedorn resonance model results and the lattice data on the equation of states. All bulk thermodynamical observables are very strongly changing with temperature when approaching the deconfinement transition. This behavior is well understood in the Hagedorn model as being due to the contribution of resonances. Although Hagedorn's model formulated for a discrete mass spectrum does not exhibit a critical behavior, it nevertheless reproduces remarkably well the lattice results in the hadronic phase. This agreement has now been extended to an analysis of fluctuations and correlations of conserved charges as well.

In summary of this section we note that a remarkably good description of lattice QCD results on the equation of states by the Hagedorn thermal model justifies, that resonances are indeed the essential degrees of freedom near deconfinement. Thus, on the thermodynamical level, modeling hadronic interactions by formation and excitation of resonances, as introduced by Hagedorn, is an excellent approximation of strong interactions.

\subsection{Resonance Gas and Heavy Ion Collisions}

Long before lattice QCD could provide a direct evidence that strong interaction thermodynamics can be quantified by the resonance gas partition function, Hagedorn's concept was verified phenomenologically by considering particle production in elementary and heavy ion collisions [34-37] (for a review, see e.g. [32]). In a strongly interacting medium, one includes the conservation of electric charge, baryon number and strangeness. In this case, the partition function of Hagedorn's thermal model depends not only on temperature but also on chemical potential $\boldsymbol{\mu}$, which guarantees, that charges are conserved on an average. For a non vanishing $\boldsymbol{\mu}$, the partition function Eq. (7.15) is replaced by

$$
\ln Z(T, V, \boldsymbol{\mu})=\sum_{i} Z_{i}^{1}(T, V, \boldsymbol{\mu})
$$

with $\boldsymbol{\mu}=\left(\mu_{B}, \mu_{S}, \mu_{Q}\right)$, where $\mu_{i}$ are the chemical potentials related to the baryon number, strangeness and electric charge conservation, respectively.

For particle $i$ carrying strangeness $S_{i}$, the baryon number $B_{i}$, the electric charge $Q_{i}$ and the spin-isospin degeneracy factor $g_{i}$, the one particle partition function, reads

$$
Z_{i}^{1}(T, V, \boldsymbol{\mu})=\frac{V g_{i} T m_{i}^{2}}{2 \pi^{2}} K_{2}\left(m_{i} / T\right) \exp \left(\frac{B_{i} \mu_{B}+S_{i} \mu_{S}+Q_{i} \mu_{Q}}{T}\right) .
$$

For $\mu=0$ one recovers the result from Eq. (7.16).

The calculation of a density $n_{i}$ of particle $i$ from the partition function Eq. (7.21) is rather straightforward [46]. It amounts to the replacement $Z_{i}^{1} \rightarrow \gamma_{i} Z_{i}^{1}$ in Eq. (7.21) 
Fig. 7.5 The ratio of the total density of positively charged pions, $n_{\pi+}^{R}$ from Eq. (7.24), and the density of thermal pions, $n_{\pi^{+}}^{\text {th }}$ from Eq. (7.23). The calculations are done in the Hagedorn resonance gas model for $\mu_{B}=250 \mathrm{MeV}$ and $\mu_{B}=550 \mathrm{MeV}$ at different temperatures

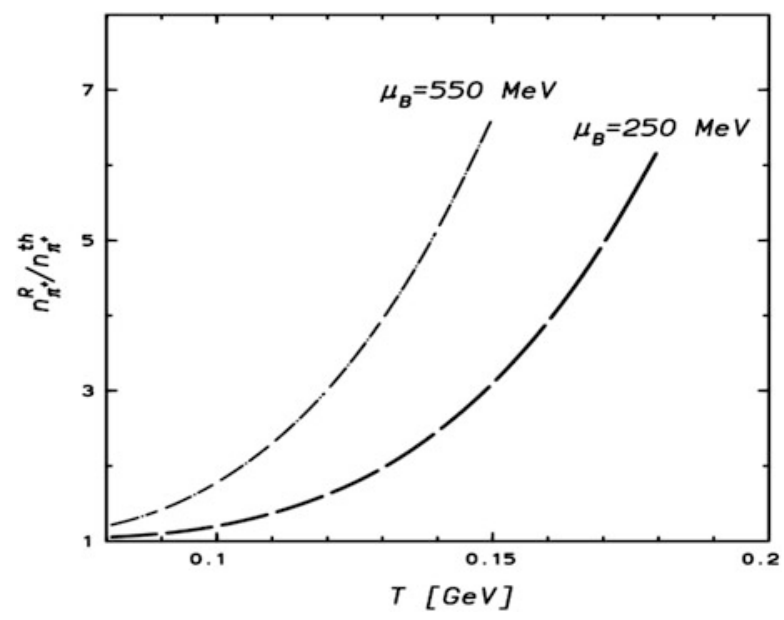

and taking a derivative with respect to the particle fugacity $\gamma_{i}$, as

$$
n_{i}=\frac{\left\langle N_{i}\right\rangle^{\text {th }}}{V}=\left.\frac{\partial \ln Z}{\partial \gamma_{i}}\right|_{\gamma_{i}=1},
$$

consequently, $n_{i}=Z_{i}^{1} / V$ with $Z_{i}^{1}$ as in Eq. (7.22).

The Hagedorn model, formulated in Eq. (7.21), describes bulk thermodynamic properties and particle composition of a thermal fireball at finite temperature and at non vanishing charge densities. If such a fireball is created in high energy heavy ion collisions, then yields of different hadron species are fully quantified by thermal parameters. However, following Hagedorn's idea, the contribution of resonances decaying into lighter particles, must be included $[2,6]$.

In Hagedorn's thermal model, the average number $\left\langle N_{i}\right\rangle$ of particles $i$ in volume $V$ and at temperature $T$ that carries strangeness $S_{i}$, the baryon number $B_{i}$, and the electric charge $Q_{i}$, is obtained from Eq. (7.21), see [2,6]

$$
\left\langle N_{i}\right\rangle(T, \boldsymbol{\mu})=\left\langle N_{i}\right\rangle^{\mathrm{th}}(T, \boldsymbol{\mu})+\sum_{j} \Gamma_{j \rightarrow i}\left\langle N_{j}\right\rangle^{t h, R}(T, \boldsymbol{\mu}) .
$$

The first term in Eq. (7.24) describes the thermal average number of particles of species $i$ from Eq. (7.23) and the second term describes overall contribution from resonances. This term is taken as a sum of all resonances that decay into particle $i$. The $\Gamma_{j \rightarrow i}$ is the corresponding decay branching ratio of $j \rightarrow i$. The multiplicities of resonances $\left\langle N_{j}\right\rangle^{\text {th, } R}$ in Eq. (7.24), are obtained from Eq. (7.23).

The importance of resonance contributions to the total particle yield in Eq. (7.24) is illustrated in Fig. 7.5 for charge pions. In Fig. 7.5 we show the ratio of the total number of charge pions from Eq. (7.24) and the number of prompt pions from Eq. (7.23). The ratio is strongly increasing with temperature and chemical potential. This is due to an increasing contribution of mesonic and baryonic resonances. From 
Eq. (7.5) it is clear, that at high temperature and/or density, the overall multiplicity of pions is mostly due to resonance decays.

The particle yields in Hagedorn's model Eq. (7.24) depend, in general, on five parameters. However, in high energy heavy ion collisions, only three parameters are independent. In the initial state the isospin asymmetry, fixes the charge chemical potential and the strangeness neutrality condition eliminates the strange chemical potential. Thus, on the level of particle multiplicity, we are left with temperature $T$ and the baryon chemical potential $\mu_{B}$ as independent parameters, as well as, with fireball volume as an overall normalization factor.

Hagedorn's thermal model introduced in Eq. (7.24) was successfully applied to describe particle yields measured in heavy ion collisions. The model was compared with available experimental data obtained in a broad energy range from AGS up to LHC. Hadron multiplicities ranging from pions to omega baryons and their ratios, as well as composite objects like e.g. deuteron or alpha particles, were used to verify if there was a set of thermal parameters $\left(T, \mu_{B}\right)$ and $V$, which simultaneously reproduces all measured yields.

The systematic studies of particle production extended over more than two decades, using experimental results at different beam energies, have revealed a clear justification, that in central heavy ion collisions particle yields are indeed consistent with the expectation of the Hagedorn thermal model. There is also a clear pattern of the energy, $\sqrt{s}$-dependence of thermal parameters. The temperature is increasing with $\sqrt{s}$, and at the SPS energy essentially saturates at the value, which corresponds to the transition temperature from a hadronic phase to a QGP, as obtained in LQCD. The chemical potential, on the other hand, is gradually decreasing with $\sqrt{s}$ and almost vanishes at the LHC.

In Fig. 7.6 we show, as an illustration, a comparison of Hagedorn's thermal model and recent data on selected particle yields, obtained by ALICE collaboration in central $\mathrm{Pb}-\mathrm{Pb}$ collisions at midrapidity at the LHC energy [38]. At such high collision energy, particle yields from Eq. (7.24) are quantified entirely by the

Fig. 7.6 Yields of several different particle species per unit rapidity normalized to spin degeneracy factor as a function of their mass. Data are from ALICE collaboration taken at the $\mathrm{LHC}$ in central $\mathrm{Pb}-\mathrm{Pb}$ collisions. The line is the Hagedorn thermal model result, Eq. (7.25), see [38]

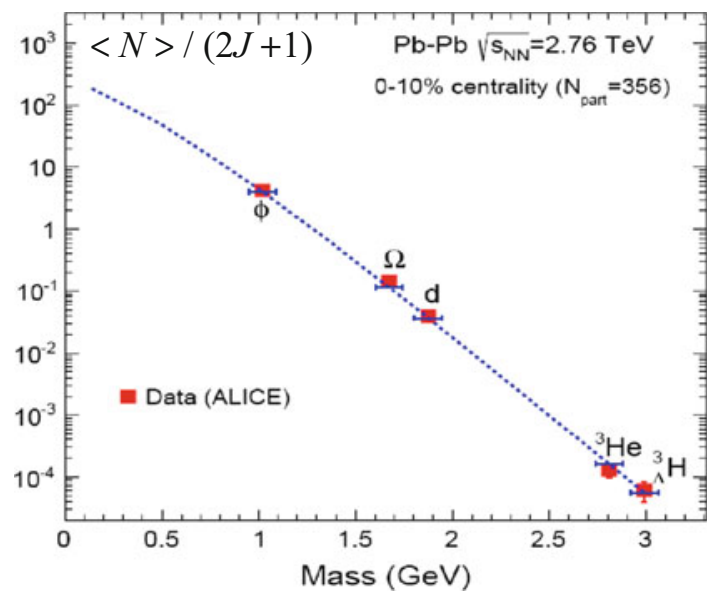


temperature and the fireball volume. ${ }^{1}$ Thus, there is transparent prediction of Hagedorn's model Eq. (7.24), that yields of heavier particles $\left\langle N_{i}\right\rangle$ with no resonance decay contributions, normalized to their spin degeneracy factor $g_{i}=(2 J+1)$, should be quantified by

$$
\frac{\left\langle N_{i}\right\rangle}{2 J+1} \simeq V T^{3}\left(\frac{m_{i}}{2 \pi T}\right)^{3 / 2} \exp \left(-m_{i} / T\right),
$$

where we have used Eq. (7.23) and the asymptotic expansion of the Bessel function, $K_{2}(x) \sim x^{-1 / 2} \exp (-x)$, valid for large $x$.

In Fig. 7.6 we show the yields of particles with no resonance contribution, like $\phi, \Omega$, the deuteron ' $\mathrm{d}$ ', ${ }^{3} \mathrm{He}$ and the hypertriton ${ }_{\Lambda}^{3} \mathrm{He}$, normalized to their spin degeneracy factor, as a function of particle mass. Also shown in this figure is the prediction from Eq. (7.25) at $T \simeq 156$ and for volume $V \simeq 5,000 \mathrm{fm}^{3}$ [38]. There is a clear coincidence of data taken in $\mathrm{Pb}-\mathrm{Pb}$ collisions at the $\mathrm{LHC}$ and predictions of the Hagedorn model Eq. (7.25). Particles with no resonance contribution measured by ALICE collaboration follow the Hagedorn's expectations that they are produced from a thermal fireball at common temperature. A similar agreement of Hagedorn's thermal concept and experimental data taken in central heavy ion collisions has been found for different yields of measured particles and collision energies from AGS, SPS, RHIC and LHC (for a review, see e.g. [32]).

\subsection{Particle Yields and Canonical Charge Conservation}

The Hagedorn thermodynamical model for particle production, was originally applied to quantify and understand particle yields and spectra measured in elementary collisions - there were no data available from heavy ion collisions.

Initial work on particle production by Hagedorn began in 1957 in collaboration with F. Cerulus when they applied the Fermi phase space model, see Sect. 25.2. In this microcanonical approach, conservation laws of baryon number or electric charge were implemented exactly. Almost 15 years later the production of complex light antinuclei, such as anti-He ${ }^{3}$, preoccupied Hagedorn [2, 6]. He realized and discussed clearly the need to find a path to enforce exact conservation of baryon number to describe the anti- $\mathrm{He}^{3}$ production correctly within the canonical statistical formulation.

Indeed, applying in $p p$ reactions the thermal model without concern for conservation of baryon number overestimates the production of anti- $\mathrm{He}^{3}$ in proton-proton collisions by seven orders of magnitude $[2,4,6]$. The reason was that when the number of particles in the interaction volume is small, one has to take into account the fact that the production of anti- $\mathrm{He}^{3}$ must be accompanied by the production

\footnotetext{
${ }^{1}$ The chemical potential $\mu$ in Eq. (7.24) vanishes, since at the LHC and at midrapidity particles and their antiparticles are produced symmetrically.
} 
of another three nucleons with energy $E_{N}$, in order to exactly conserve the baryon number. Thus, in case the production of anti- $\mathrm{He}^{3}$ is not originating from reservoir of many antiquarks or antinucleons already present in a large volume, but is rather originating from some small volume $V_{p p}$ that is present in $p p$ collisions, the abundance of anti- $\mathrm{He}^{3}$ will not be proportional to the single standard Boltzmann factor, as in Eq. (7.25)

$$
n_{\overline{\mathrm{He}}^{3}} \sim \exp \left(-m_{\overline{\mathrm{He}}^{3}} / T\right)
$$

but is accompanied by additional Boltzmann factors that characterize the production of the associated nucleons, needed in order to conserve baryon number $[2,6]$

$$
n_{\overline{\mathrm{He}}^{3}} \sim \exp \left(-m_{\overline{\mathrm{He}}^{3}} / T\right)\left[V_{\mathrm{pp}} \int \frac{d^{3} p}{(2 \pi)^{3}} \exp \left(-\frac{E_{N}}{T}\right)\right]^{3} .
$$

This suppresses the rate and introduces a strong power-law dependence on volume $V_{p p}$ for the anti-He $\mathrm{H}^{3}$ yield.

The problem of exact conservation of discrete quantum numbers in a thermal model formulated in early 1970s by Hagedorn in the context of baryon number conservation remained unsolved for a decade. When the heavy ion QGP research program was approaching and strangeness emerged as a potential QGP signature, Hagedorn pointed out the need to consider exact conservation of strangeness (Rafelski, private communication). This is the reason that the old problem of baryon number conservation was solved in the new context of strangeness conservation [39-41], see also Sect.27.6. A more general solution, applicable to all discrete conserved charges, abelian and non-abelian, was also introduced in [42] and expanded in [43-48]. Recently, it has become clear that a similar treatment should be followed not only for strangeness but also for charm abundance study in high energy $e^{+} e^{-}$collisions $[49,50]$.

To summarize this section, we note that the usual form of the statistical model, based on a grand canonical formulation of the conservation laws, cannot be used when either the temperature or the volume or both are small. As a rough estimate, one needs $V T^{3}>1$ for a grand canonical description to hold [39, 46]. In the opposite limit, a path was found within the canonical ensemble to enforce charge conservations exactly.

The canonical approach has been shown to provide a consistent description of particle production in high energy hadron-hadron, $e^{+} e^{-}$and peripheral heavy ion collisions [32, 45, 49, 50]. As noted in the context of developing strangeness as signature of QGP, see Sect.27.6, such a model also provides, within the realm of assumed strangeness chemical equilibrium, a description of an observed increase of single- and multi-strange particle yields from $p p, p A$ to $A A$ collisions and its energy dependence [40]. 


\title{
7.7 Concluding Remarks
}

Rolf Hagedorn's work, introducing concepts from statistical mechanics and from the mathematics of self-similarity into the analysis of high energy multiparticle production, started a new field of research, alive and active still today. On the theory side, the limiting temperature of hadronic matter and the behavior of the Hagedorn resonance gas approaching that limit were subsequently verified by first principle calculations in finite temperature QCD. On the experimental side, particle yields as well as, more recently, fluctuations of conserved quantities, were also found to follow the pattern predicted by the Hagedorn resonance gas. Rarely has an idea in physics risen from such humble and little appreciated beginnings to such a striking vindication. So perhaps it is appropriate to close with a poetic summary one of us (HS) formulated some 20 years ago for a Hagedorn-Fest, with a slight update.

\section{HOT HADRONIC MATTER}

(A Poetic Summary)

\section{In days of old}

a tale was told

of hadrons ever fatter.

Behold, my friends, said Hagedorn, the ultimate of matter.

\author{
Then Muster Mark \\ called in the quarks, \\ to hadrons they were mated. \\ Of colors three, and never free, \\ all to confinement fated. \\ But in dense matter, \\ their bonds can shatter \\ and they freely move around. \\ Above $T_{\mathrm{H}}$, their colors shine \\ as the QGP is found. \\ Said Hagedorn, \\ when quarks were born \\ they had different advances. \\ Today they form, as we can see, \\ a gas of all their chances.
}


Open Access This book is distributed under the terms of the Creative Commons Attribution Noncommercial License which permits any noncommercial use, distribution, and reproduction in any medium, provided the original author(s) and sources are credited.

\section{References}

1. R. Hagedorn, Thermodynamics of distinguishable particles: A key to high-energy strong interactions?, see Chapter 19, and Commentary by J. Rafelski Chapter 18, in this volume.

2. R. Hagedorn, Statistical thermodynamics of strong interactions at high-energies. Nuovo Cimento Suppl. 3, 147 (1965)

3. R. Hagedorn, J. Ranft, Statistical thermodynamics of strong interactions at high-energies, 2. Momentum spectra of particles produced in $p p$ collisions. Nuovo Cimento Suppl. 6, 169 (1968)

4. R. Hagedorn, Statistical thermodynamics of strong interactions at high energies, 3. Heavy-pair (quark) production rates. Nuovo Cimento Suppl. 6, 311 (1968)

5. R. Hagedorn, Hadronic matter near the boiling point. Nuovo Cimento A 56, 1027 (1968)

6. R. Hagedorn, Thermodynamics of strong interactions. CERN-Report 71-12 (1971)

7. G.H. Hardy, S. Ramanujan, Asymptotic formulae in combinatory analysis. Proc. Lond. Math. Soc. 17, 75 (1918)

8. Ph. Blanchard, S. Fortunato, H. Satz, The Hagedorn temperature and partition thermodynamics. Eur. Phys. J. C 34, 361 (2004)

9. W. Nahm, Analytical solution of the statistical bootstrap model. Phys. B 45, 525 (1972)

10. G. Veneziano, Construction of a crossing - symmetric, Regge behaved amplitude for linearly rising trajectories. Nuovo Cimento A 57, 190 (1968)

11. K. Bardakci, S. Mandelstam, Analytic solution of the linear-trajectory bootstrap. Phys. Rev. 184, 1640 (1969)

12. S. Fubini, G. Veneziano, Level structure of dual-resonance models. Nuovo Cimento A 64, 811 (1969)

13. E. Beth, G.E. Uhlenbeck, The quantum theory of the non-ideal gas, II. Behaviour at low temperatures. Physica 4, 915 (1937)

14. R. Dashen, S.-K. Ma, H.J. Bernstein, S Matrix formulation of statistical mechanics. Phys. Rev. 187, 345 (1969)

15. N. Cabibbo, G. Parisi. Exponential hadronic spectrum and quark liberation. Phys. Lett. B 59, 67 (1975)

16. H. Satz, Critical behavior of hadronic matter, 1. critical point exponents. Phys. Rev. D19, 1912 (1979)

17. M.I. Gorenstein, V.A. Miransky, V.P. Shelest, G.M. Zinovev, H. Satz, The physical content of the statistical bootstrap. Nucl. Phys. B76, 453 (1974)

18. I.Ya. Pomeranchuk, On the theory of multiple particle production in a single collision. Dokl. Akad. Nauk 78, 889 (1951)

19. G. Baym, Confinement of quarks in nuclear matter. Phys. A 96, 131 (1979)

20. T. Çelik, F. Karsch, H. Satz, A percolation approach to strongly interacting matter. Phys. Lett. B 97, 128 (1980)

21. H. Satz, Extreme states of matter in strong interaction physics: An Introduction. Lect. Notes Phys. 841 (2012)

22. (a) L.D. McLerran, B. Svetitsky, A Monte Carlo study of SU(2) Yang-Mills theory at finite temperature. Phys. Lett. B 98, 195 (1981); (b) J. Kuti, J. Polonyi, K. Szlachanyi, Monte Carlo study of SU(2) Gauge theory at finite temperature. Phys. Lett. B 98, 199 (1981); (c) J. Engels, F. Karsch, I. Montvay, H. Satz, High temperature SU(2) gluon matter on the lattice. Phys. Lett. B 101, 89 (1981)

23. F. Karsch, Lattice QCD at high temperature and density. Lect. Notes. Phys. 583, 209 (2002)

24. Y. Aoki, G. Endrodi, Z. Fodor, S.D. Katz, K.K. Szabo, The Order of the quantum chromodynamics transition predicted by the standard model of particle physics. Nature 443, 675 (2006) 
25. A. Bazavov et al. [HotQCD], Equation of state in (2+1)-flavor QCD. Phys. Rev. D 90, 094503 (2014)

26. S. Ejiri, F. Karsch, K. Redlich, Hadronic fluctuations at the QCD phase transition. Phys. Lett. B 633, 275 (2006)

27. A. Bazavov et al. [HotQCD Collaboration], Fluctuations and Correlations of net baryon number, electric charge, and strangeness: A comparison of lattice QCD results with the hadron resonance gas model. Phys. Rev. D 86, 034509 (2012)

28. A. Bazavov et al., Additional strange hadrons from QCD thermodynamics and strangeness freezeout in heavy ion collisions. Phys. Rev. Lett. 113, 072001 (2014)

29. (a) F. Karsch, K. Redlich, A. Tawfik, Thermodynamics at nonzero baryon number density: A Comparison of lattice and hadron resonance gas model calculations. Phys. Lett. B 571, 67 (2003); (b) F. Karsch, K. Redlich, A. Tawfik, Hadron resonance mass spectrum and lattice QCD thermodynamics. Eur. Phys. J. C 29, 549 (2003)

30. F. Karsch, Thermodynamics of strong interaction matter from lattice QCD and the hadron resonance gas model. Acta Phys. Pol. Suppl. 7, 117 (2014)

31. K.A. Olive et al. [Particle Data Group], Chin. Phys. C 38, 090001 (2014)

32. P. Braun-Munzinger, K. Redlich, J. Stachel, Particle production in heavy ion collisions, in Quark Gluon Plasma 2, ed. by R.C. Hwa (World Scientific, Singapore, 2004), p. 491

33. T. Bhattacharya et al., QCD phase transition with chiral quarks and physical quark masses. Phys. Rev. Lett. 113, 082001 (2014)

34. J. Cleymans, H. Satz, Thermal hadron production in high-energy heavy ion collisions. Z. Phys. C 57, 135 (1993)

35. K. Redlich, J. Cleymans, H. Satz, E. Suhonen, Hadronization of quark - gluon plasma. Nucl. Phys. A 566, 391 (1994)

36. P. Braun-Munzinger, I. Heppe, J. Stachel, Chemical equilibration in $\mathrm{Pb}+\mathrm{Pb}$ collisions at the SPS. Phys. Lett. B 344, 43 (1995)

37. F. Becattini, A Thermodynamical approach to hadron production in e+e- collisions. Z. Phys. C 69, 485 (1996)

38. (a) A. Andronic, An overview of the experimental study of quark-gluon matter in highenergy nucleus-nucleus collisions. Int. J. Mod. Phys. A 29, 1430047 (2014); (b) J. Stachel, A. Andronic, P. Braun-Munzinger, K. Redlich Confronting LHC data with the statistical hadronization model. J. Phys. Conf. Ser. 509, 012019 (2014)

39. J. Rafelski, M. Danos, The importance of the reaction volume in hadronic collisions. Phys. Lett. B 97, 279 (1980)

40. S. Hamieh, K. Redlich, A. Tounsi, Canonical description of strangeness enhancement from p-A to $\mathrm{Pb}-\mathrm{Pb}$ collisions. Phys. Lett. B 486, 61 (2000)

41. J. Cleymans, H. Oeschler, K. Redlich, Influence of impact parameter on thermal description of relativistic heavy ion collisions at (1-2) A-GeV. Phys. Rev. C 59, 1663 (1999)

42. K. Redlich, L. Turko, Phase transitions in the statistical bootstrap model with an internal symmetry. Z. Phys. C 5, 201 (1980)

43. L. Turko, Quantum gases with internal symmetry. Phys. Lett. B 104, 153 (1981)

44. M.I. Gorenstein, V.K. Petrov, G.M. Zinovev, Phase transition in the Hadron gas model. Phys. Lett. B 106, 327 (1981)

45. B. Muller, J. Rafelski, Role of internal symmetry in $p \bar{p}$ annihilation. Phys. Lett. B 116, 274 (1982)

46. R. Hagedorn, K. Redlich, Statistical thermodynamics in relativistic particle and ion physics: Canonical or grand canonical? Z. Phys. C 27, 541 (1985)

47. C. Derreth, W. Greiner, H.T. Elze, J. Rafelski, Strangeness abundances in anti-p nucleus annihilations. Phys. Rev. C 31, 1360 (1985)

48. H.T. Elze, W. Greiner, J. Rafelski, Color degrees of freedom in a Quark - Glue plasma at finite Baryon density. Z. Phys. C 24, 361 (1984)

49. F. Becattini, P. Castorina, J. Manninen, H. Satz, The thermal production of strange and nonstrange hadrons in e+ e- collisions. Eur. Phys. J. C 56, 493 (2008)

50. A. Andronic, F. Beutler, P. Braun-Munzinger, K. Redlich, J. Stachel, Thermal description of hadron production in e+e- collisions revisited. Phys. Lett. B 675, 312 (2009) 